\title{
Investigation of the correlation between radial depth of cut (RDOC) and axial depth of cut (ADOC) in NX-CAM system: Simulation Studies \\ Mohamad Hafiz ${ }^{1}$ and M.N.Osman Zahid ${ }^{1 *}$
}

${ }^{1}$ Faculty of Manufacturing Engineering, Universiti Malaysia Pahang, 26660, Pekan, Pahang, Malaysia

\begin{abstract}
This paper outlines a simulation study to investigate the correlation between radial depth of cut (RDOC) and axial depth of cut (ADOC) in 4 axis machining processes. Computer-aided Manufacturing (CAM) plays a crucial role in simulating cutting operations before the real process executed. Several cutting parameters can be analysed through effective CAM program to predict the outcomes. However, an efficient program required skilled operator to develop the process planning. Besides, a standard CAM system has limited capabilities to handle repetitive simulation due to unavailable function to support the analysis. In this study, the combination of RDOC and ADOC is analyses on different parts features consist of planar surface and non-planar surface. Several set of pairing percentages between RDOC and ADOC are used in the simulation program to analyses the cutting operations. In order to perform the simulation, a customized program was developed in NX CAM system to assist the routines. It capable to generate machining data files from each simulation and assess the total volume removed. A pairing set with high volume removal is denoted as an optimum value and will be chosen as a cutting parameter in finishing operation. Generally, ADOC has a significant effect compared to RDOC in terms of total volume removed from the workpiece.
\end{abstract}

\section{Introduction}

An optimum selection of cutting parameter is highly contributes to the increasing of productivity and decreasing of production cost. Nowadays, cutting parameter simulation is essential for manufacturing sector to survive in competitive market. In machining processes, simulation of cutting parameter is considered to be a significant tool for improvement to obtain a good quality and reduce the overall process planning time [1]. In order to obtain higher geometrical accuracy with good surface finish, most of parts are developed by using CNC machine technology. In machining processes, the amount of volume removed from raw material is considerable to produce the desired geometrical (Radhika 2015). In addition to that, CNC machining processes is well known as more expensive rather than others manufacturing processes such as forging or casting. Therefore, because of $\mathrm{CNC}$ machining required high capital cost, economic processes is needed in order to ensure return of investment. Generally, optimal machining operation is mainly depend on the proper selection of cutting parameter such as cutting speed, feed and depth of cut. The machining operation should be aimed to increase the quality of the product surface at minimum machining time and simple process planning. In order to achieve these objectives, machining simulation program is required to assist machinist to produce and select an optimum cutting parameter for the operations. The purpose of simulation is to provide early information and capable to identify the issue that will happen during machining processes [2].

\section{Customization of CAD/CAM systems}

A digital management program called "Unified Digitization System" has been developed by Shao Wei (2014) for the part parameters based on UG (Unigraphics $\mathrm{NX)}$ platform [3]. The program was developed to improvise the existing digital data management system where it is inconsistent in terms of product design data, manufacturing information and detection of digital plant construction. Nowadays, modern industries require a digital management platform that capable to storing uniform data related to product design, process design and manufacturing processes. Therefore, this system is developed to provide solutions by combining all digital information in a single database. SQL server database was established for database management in order to synchronize all the parts parameters information in design, manufacture and detection. Fig. 1 illustrates the structure of digital management system and inspection planning. Databases management consists of 3 major components which are product design, information collection and inspection planning. NX software and C\# is the main development platform used to create product design, also to build an application for inspection planning and information collection algorithm.

Palekar (2014) has developed a Master Model application to improvise the updating process for assembly models [4]. The application was developed to address the problems associated with the parts assembly in NX CAM systems. The information updating process for parts consist of hundreds sub-assemblies components is a very difficult task in the modern CAD/CAM industry. A slightly mistake while updating the parts information (parameter, dimension and etc.) can lead to error in the overall assembly model. Additionally, it will

*nafis@ump.edu.my 
indirectly affect the overall time consumed, requires a high graphic and high probability to cause system memory error (out of memory, memory access violation and etc.). The Application Programming Interfaces (API) has been used to develop the proposed application within Microsoft Visual Studio. For programming purpose, the combination of Journaling Tools and VB.net has been employed. The user will enter the necessary input into developed application, and then the input will be transferred to NX through excel files. The application will run the update process on the NX assembly model in background mode. After that, the application will generate an error report, if any error occurs during the assembly model updating processes. Through error report, the part that has an error could be identified and can be modified individually later. The Master Model application manage to reduce the product life cycle time and percentage of error generation during NX parts assembly information updating processes.

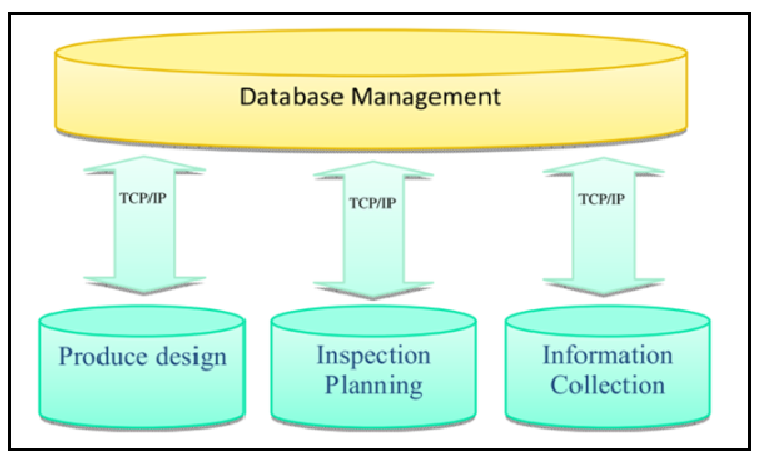

Fig. 1. The structure of digital management system and inspection planning [3]

Nowadays, almost all the constructional process for products design is aided by using $\mathrm{CAD} / \mathrm{CAM}$ systems. Each of designing processes has natural routines tasks that are possible to be automated. Zbiciak (2015) worked out an application to automate the engineering task in NX CAM environment that used to generate $3 \mathrm{D}$ design for gear wheel model called, The Generator Module (Fig. 2). The main function of the module is to develop and modelling the spur and helical gears. There has two types of validation test, the first test is to validate the design parameter calculation and second test is validating the geometry involutes between proposed with the standard approach. The program was developed by using NX modelling function by manipulating the vector graphics to generate a journaling code for customization. According to the simulation results, the generator module manage to facilitates the constructional design process with minimum time and process planning. The main advantage of the application is it capable to reduce the modelling time compared to conventional methods [5].

Gawai (2015) develop customization software to design a solid shaft coupling assembly model by using Unigraphics (NX) and Knowledge-Fusion (KF) Programming [6]. Knowledge-Fusion (KF) is an integrated tools that permits the NX end user to extend the NX capabilities by increasing the design speed and intelligently control the own functions as needed by the developer [7]. KF supports all the NX functions that are used to automate the required processes. An Open User Interfaces styler was utilized to develop and create a dialog box for NX user interfaces as shown in Fig. 3. The customized software used to automate the design process for shaft coupling modelling, where it was managed to decrease the processing time and effectively simplify the modelling process planning.

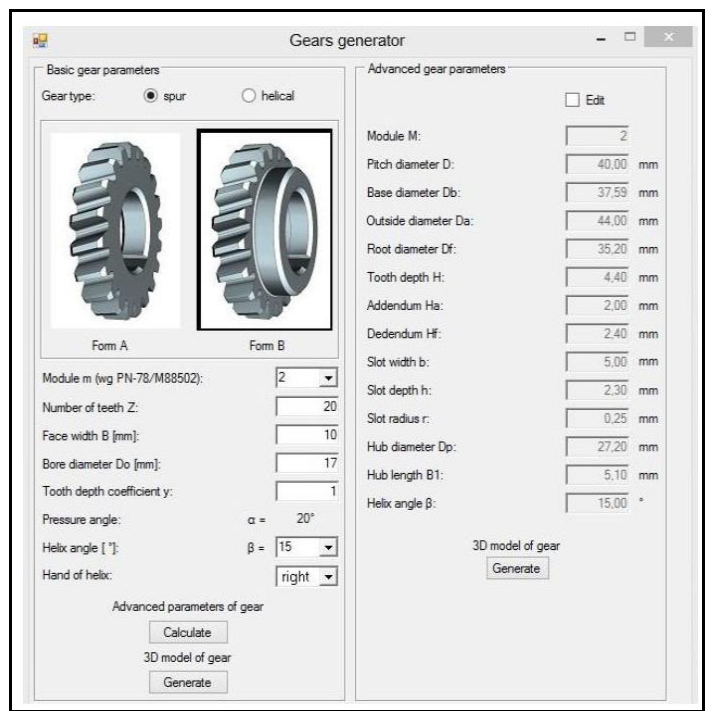

Fig. 2. The Generator Module GUI [5]

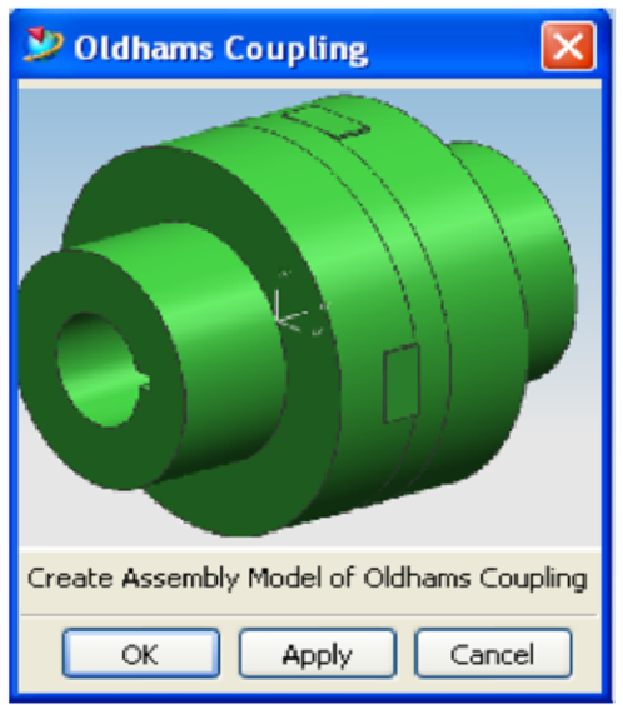

Fig. 3. User Interface of Coupling Assembly [6]

In CAD modelling, the modification processes requires a repetitive procedure to get the desired model. Instead of that, there is another way to automate routine while constraining several constant parameters. Siddesh (2015) has introduced an automation method to generate the CAD models in CATIA systems by using 
programming application [8]. In general, automation generation of CAD modelling can be executed in two methods. First method, the coding was written manually then uploads to the program while second method is utilizing macro tool that available in CAD/CAM system. Manual program used a user form interface that has been developed in visual basic application (VBA). It has only two buttons, save and open. The save button is used to store the parameters, while the open button is used to edit the parameters. However, manual method is very vulnerable to errors due to the coding was written manually before uploading into the application. This method is also complicated and takes a long time for repeating processes. Therefore, the author develops a program to implement the automation process for CAD generation. Standard user form was used as an interface in macro method and able to help user to interact with CAD model. It contains a labeled text box and also a commands button to start the design process. The user just needs to enter the value into the box then press "create spring" button. Using the proposed system method, the time required to generate the CAD model for repetitive work can be reduced up to $90 \%$. In addition, the proposed method also improves the consistency of the design model and reduces the probability of human error.

Benaouali (2017) develop a procedures to automate the integration between Computer-Aided Design (CAD) and Computer-Aided Engineering (CAE), which is used to make a design and analysis on aircraft wings [9]. The proposed procedures are developed with the use of Graphic Interactive Programming (GRIP) tool automation that available in the Siemens NX software. The main purpose of the integration is to eliminate the repetitive cost effort and to simplify the optimization procedure. During the aircraft model optimization phase, there is a several data changing in design, geometric and analysis parameters. The model needs to be updated for each parameter changing. This task will be complicated and time consuming if performed manually, particularly in the case of model that have a few number of parameter. Therefore, it is requisite to integrate $\mathrm{CAD}$ and CAE tools as well as automate the integration procedure. The GRIP program was employed to handle the modelling parameters data and send the compiled information to Siemens NX software. For the analysis parameters, PATRAN software was used to send the information to NASTRAN software. PATRAN and NASTRAN is the post-processing software that use for Finite Element Analysis (FEA), which is providing meshing and analysis setup for solid modelling. Once all the procedures setup and analysis was done, the result file is produced in .f06 format and will be extracted by necessary application to generate the desired results.

Nafis (2017) has developed a machining simulation programs to improvise the manufacturing process planning for CNC Rapid Manufacturing (CNC-RM) applications [10]. This simulation tool is intended to apply the automation approach to handle machining process planning. In principle, planning task in the machining operation is correlated with machinist experienced, skill and time. A few improvements in CAM technology was established to assist minimum skill user to build a machining operation. A customized program has been developed using visual basic programming and incorporated with $\mathrm{NX}$ platform. It equipped with simple Graphical User Interfaces (GUI) that has a few inputs to be defined by the user. The program was simulated with several models that have varied properties in terms of geometry and size, to validate the program stability level. The simulation results has shown that the developed program manages to build a complete machining operation and successfully minimizes the time spent for process planning up to $79 \%$ compared to conventional approach.

\section{Methodologies}

\subsection{Code Customization and GUI development}

In this study, NX CAM system has been used as a platform to develop customized simulation program for machining operation, called 'FinishSimulCAM'. In order to develop a simulation program, all the machining task and operation must be execute manually at first and recorded using journaling function. Journaling is a rapid automation tools that used to records, edit and replay all interactive in NX sessions [6]. It will produces a Visual Basic scripts files which can be used to develop a customize program based on user requirement. The scripts can be edited and enhanced using simple programming coding to construct a new program. It also capable to be integrated with a graphical user interfaces (GUI) which can be used to generate a program for machining operation. In this study, several methods have been adopted to develop a more systematic and user-friendly simulation program as shown in Fig. 4.

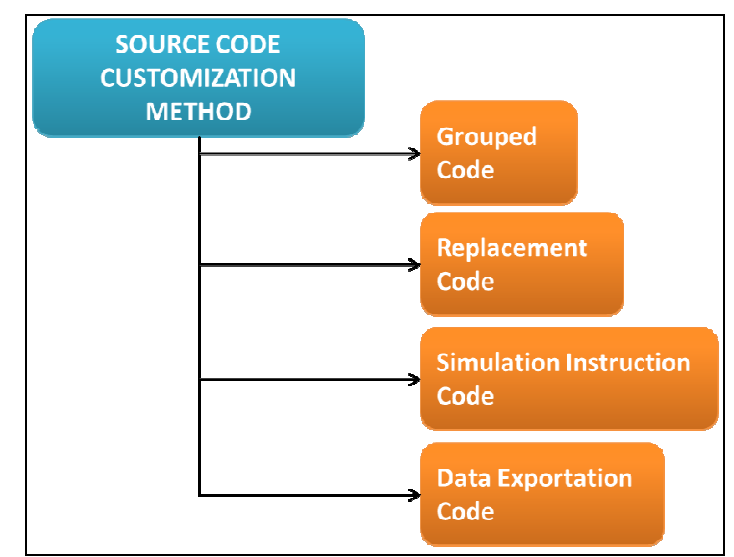

Fig. 4. Source Code Customization Method

In general, four customization techniques are used, namely Grouped Code, Replacement Code, Simulation Instruction Code and Data Exportation Code. Grouped Code techniques are used to unify all input that share the same value with a single variable and embedded in the FinishSimulCAM. Therefore, a parameter value from 
user input can be used for many functions behind the simulation program code such as, workpiece diameter value that used to determine the maximum cutting range depth and extension cutting area distance. Replacement Code techniques are used to remove the code stickiness from original source code by replacing the code that refers to specific objects with the pop-up selection window. With this modification, it will increase the adaptability of FinishSimulCAM program with different parts or model that will be used in the machining simulation analysis.

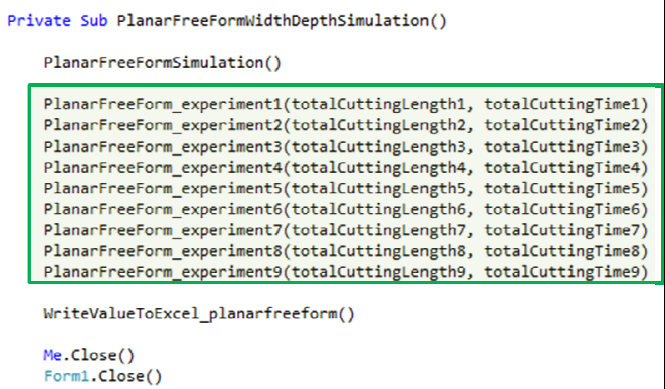

Fig. 5. Simulation Instruction Code

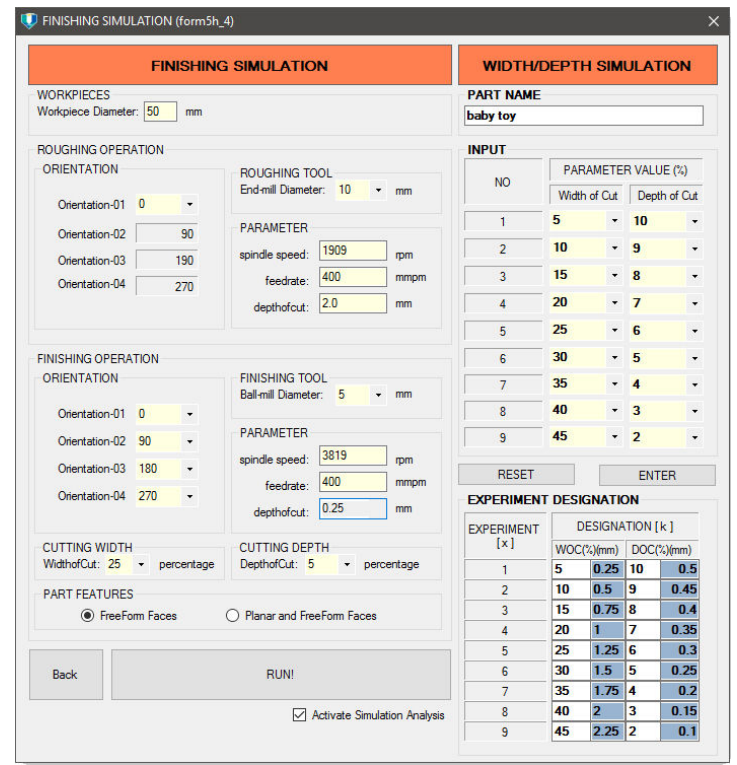

Fig. 6. FinishSimulCAM GUI

The Simulation Instruction Code techniques are used in the code customization procedure to automate the repetitive operation and ensure the simulation analyses are continuously run. Having this code, the FinishSimulCAM program will repeat the machining simulation analysis with the predefined parameter set without requiring user intervention between the processes. Therefore, the simulation process will be more efficient and will produce more accurate analysis results with fully automatic procedures between the processes. Fig. 5 shows the simulation instruction code embedded inside FinishSimulCAM program. Another customization that used in FinishSimulCAM program is Data Exportation Code technique. This code is used to record and publish all the information regarding to machining parameter and conclude the results in Excel files format. Once all the code customizations are completely embedded inside the source code, these codes will be incorporated with a graphical user interface (GUI). Graphical User interface (GUI) allow the user to interact with the program where a specific function and control are embedded inside the program through visual indicators [11]. Fig. 6 shows the FinishSimulCAM GUI that has been developed to execute the machining simulation in this study.

\subsection{Machining simulation and verification}

In this study, machining simulations were performed through FinishSimulCAM program, where consists of roughing and finishing operation. In order to optimize the volume removed, roughing operation were carried out through several cutting angle with four number of orientations [12]. Cutting orientations which proposed highest volume removed is denoted as an optimum and will be used in the roughing operation. Meanwhile, in finishing operation, the cutting angle and number of orientations are defined based on parts geometric.

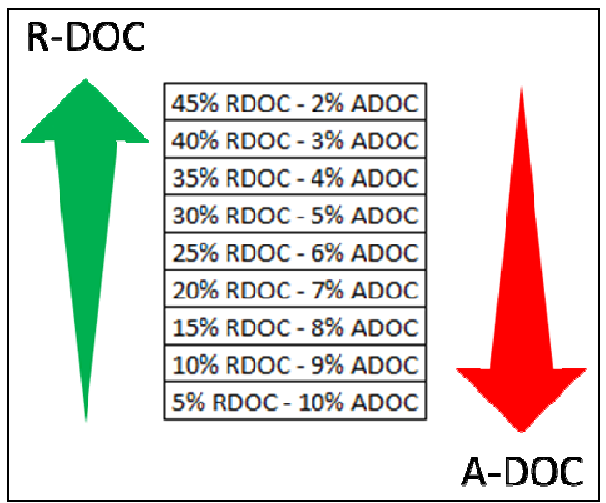

Fig. 7. Pairing Parameter of RDOC and ADOC

Generally, this study attempts to simulate the correlations of cutting parameter between Radial depthof-cut (RDOC) and Axial depth-of-cut (ADOC). Radial depth of cut (RDOC) can be defined as a distance of tool stepping over into machined parts, where Axial depth of cut (ADOC) is a distance of tool step down into a machined parts. Several pairing set of parameter in percentage value were used as an input for finishing operation. Range of parameter value is between $2 \%$ to $10 \%$ of cutter diameter for ADOC, whereas $5 \%$ to $45 \%$ of cutter diameter for RDOC. Fig. 7 shows the set of parameter used in the simulation. Each pairing set is evaluated based on total volume removed. The simulation operations are executed in NX 10 platform. A series of machining operation were generated through the simulation processes and the FinishSimulCAM used to capture the information and published the required data in excel and cutter location source (.clsf) files format. In order to analyse and calculate the volume removed, CGTech VERICUT 8.0 [13] was used since 
this software can be directly integrated within NX CAM interface through .clsf files.

\section{Results and Discussions}

The machining simulations have been executed using three different models: toy jack, alphabet $\mathrm{S}$ and salt bottle. Generally, roughing and finishing operations are executed through several cutting orientations based on $4^{\text {th }}$ axis machining. During finishing process, two opposite orientations are selected to simulate the process with the proposed combination of RDOC and ADOC. The main evaluation criterion is volume removed. Table 1, Table 2 and Table 3 summarized the result that has been produced from the machining simulations based on different machined parts. Referring to the table, 9 experiments were performed with different pair of $\mathrm{ADOC}$ and RDOC parameter values in percentage. The amount of volume removed was measured in millimetre cubic $\left(\mathrm{mm}^{3}\right)$ and presented in the last column of the table. Referring to the table, an experiment that highlighted with orange colour indicates the results of minimum volume removed, blue colour indicates median volume removed and green colour indicates maximum volume removed. It is important to be noted that all the machining parameter were set to constant for all experiment executed along the simulations. Therefore, the results gained from the simulations are totally relied on performance of RDOC and ADOC parameter.

Table 1. Simulation results for Toy Jack

\begin{tabular}{|c|c|c|c|c|}
\hline \multirow{5}{*}{ Model } & Exp. & $\begin{array}{c}\text { Radial } \\
\text { Depth } \\
\text { of Cut } \\
\text { (RDOC) } \\
(\%)\end{array}$ & $\begin{array}{c}\text { Axial } \\
\text { Depth } \\
\text { of Cut } \\
(\text { ADOC) } \\
(\%)\end{array}$ & $\begin{array}{c}\text { Volume } \\
\text { Removed } \\
\left(\mathrm{mm}^{3}\right)\end{array}$ \\
\hline \multirow{6}{*}{$\begin{array}{c}\text { Toy } \\
\text { Jack }\end{array}$} & Ex1 & 5 & 10 & $152,689.36$ \\
\cline { 2 - 5 } & Ex2 & 10 & 9 & $152,847.52$ \\
\cline { 2 - 5 } & Ex3 & 15 & 8 & $152,954.47$ \\
\cline { 2 - 5 } & Ex4 & 20 & 7 & $152,920.80$ \\
\cline { 2 - 5 } & Ex5 & 25 & 6 & $152,932.29$ \\
\cline { 2 - 5 } & Ex6 & 30 & 5 & $152,875.15$ \\
\cline { 2 - 5 } & Ex7 & 35 & 4 & $153,010.08$ \\
\cline { 2 - 5 } & Ex8 & 40 & 3 & $152,984.69$ \\
\cline { 2 - 5 } & Ex9 & 45 & 2 & $153,059.45$ \\
\hline
\end{tabular}

Table 2. Simulation results for Alphabet S

\begin{tabular}{|c|c|c|c|c|}
\hline \multirow{5}{*}{ Model } & Exp. & $\begin{array}{c}\text { Radial } \\
\text { Depth } \\
\text { of Cut } \\
\text { (RDOC) } \\
(\%)\end{array}$ & $\begin{array}{c}\text { Axial } \\
\text { Depth } \\
\text { of Cut } \\
(\text { ADOC }) \\
(\%)\end{array}$ & $\begin{array}{c}\text { Volume } \\
\text { Removed } \\
\left(\mathrm{mm}^{3}\right)\end{array}$ \\
\hline \multirow{5}{*}{$\begin{array}{c}\text { Alphabet } \\
\text { S }\end{array}$} & Ex1 & 5 & 10 & $165,099.77$ \\
\cline { 2 - 5 } & Ex2 & 10 & 9 & $165,193.82$ \\
\cline { 2 - 5 } & Ex3 & 15 & 8 & $165,279.80$ \\
\cline { 2 - 5 } & Ex4 & 20 & 7 & $165,207.07$ \\
\cline { 2 - 5 } & Ex5 & 25 & 6 & $165,300.04$ \\
\cline { 2 - 5 } & Ex6 & 30 & 5 & $165,326.59$ \\
\cline { 2 - 5 } & Ex7 & 35 & 4 & $165,371.95$ \\
\cline { 2 - 5 } & Ex8 & 40 & 3 & 165.426 .69 \\
\cline { 2 - 5 } & Ex9 & 45 & 2 & $165,412.91$ \\
\hline
\end{tabular}

Table 3. Simulation results for Salt Bottle

\begin{tabular}{|c|c|c|c|c|}
\hline \multirow{5}{*}{ Model } & Exp. & $\begin{array}{c}\text { Radial } \\
\text { Depth } \\
\text { of Cut } \\
\text { (RDOC) } \\
(\%)\end{array}$ & $\begin{array}{c}\text { Axial } \\
\text { Depth } \\
\text { of Cut } \\
(\text { ADOC) } \\
(\%)\end{array}$ & $\begin{array}{c}\text { Volume } \\
\text { Removed } \\
\left(\mathrm{mm}^{3}\right)\end{array}$ \\
\hline \multirow{5}{*}{$\begin{array}{c}\text { Salt } \\
\text { Bottle }\end{array}$} & Ex1 & 5 & 10 & $121,291.44$ \\
\cline { 2 - 5 } & Ex2 & 10 & 9 & $121,253.38$ \\
\cline { 2 - 5 } & Ex3 & 15 & 8 & $121,182.56$ \\
\cline { 2 - 5 } & Ex4 & 20 & 7 & $121,215.24$ \\
\cline { 2 - 5 } & Ex5 & 25 & 6 & $121,303.95$ \\
\cline { 2 - 5 } & Ex6 & 30 & 5 & $121,292.98$ \\
\cline { 2 - 5 } & Ex7 & 35 & 4 & $121,271.52$ \\
\cline { 2 - 5 } & Ex8 & 40 & 3 & $121,307.80$ \\
\cline { 2 - 5 } & Ex9 & 45 & 2 & 121.265 .55 \\
\hline
\end{tabular}

After machining simulation were executed using 3 different models, the findings show a trend where highest volume removed is achieved by using high RDOC and low ADOC parameter. Machining simulation for Toy Jack models shows the highest volume removed is achieved in experiment $9(45 \%$-RDOC and $2 \%$ $\mathrm{ADOC})$, the median volume removed achieved in experiment $5(25 \%$-RDOC and 6\%-ADOC) and lowest volume removed achieved in experiment 1 (5\%-RDOC and 10\%-ADOC). For Alphabets S models, the highest volume removed is achieved in experiment $8(40 \%$ RDOC and 3\%-ADOC) where median and lowest volume removed same as Toy Jack model. For Salt Bottle, the highest volume removed achieved in experiment 8 (40\%-RDOC and 3\%-ADOC), median volume removed achieved in experiment 7 (35\%-RDOC and 4\%-ADOC) and lowest volume removed achieved in experiment 3 (15\%-RDOC and 8\%-ADOC). Furthermore, in order to visualize the implications of pairing parameter towards volume removed, the material distribution assessment was produced and excess volume calculated using VERICUT 8.0 software. Table 4, 5 and 6 shows the comparison of material distribution diagram for minimum and maximum volume removed. It can be clearly seen that the experiment using higher RDOC with lower ADOC removed more material than experiment using lower RDOC with higher ADOC.

Table 4. Material distribution assessment for Toy Jack

\begin{tabular}{|c|c|}
\hline \multicolumn{2}{|c|}{ Toy Jack } \\
\hline Experiment 1 & Experiment 9 \\
\hline & \\
& \\
Excess Volume: & \\
$66.65 \mathrm{~mm}^{3}$ & Excess Volume: \\
& $62.42 \mathrm{~mm}^{3}$ \\
\hline
\end{tabular}



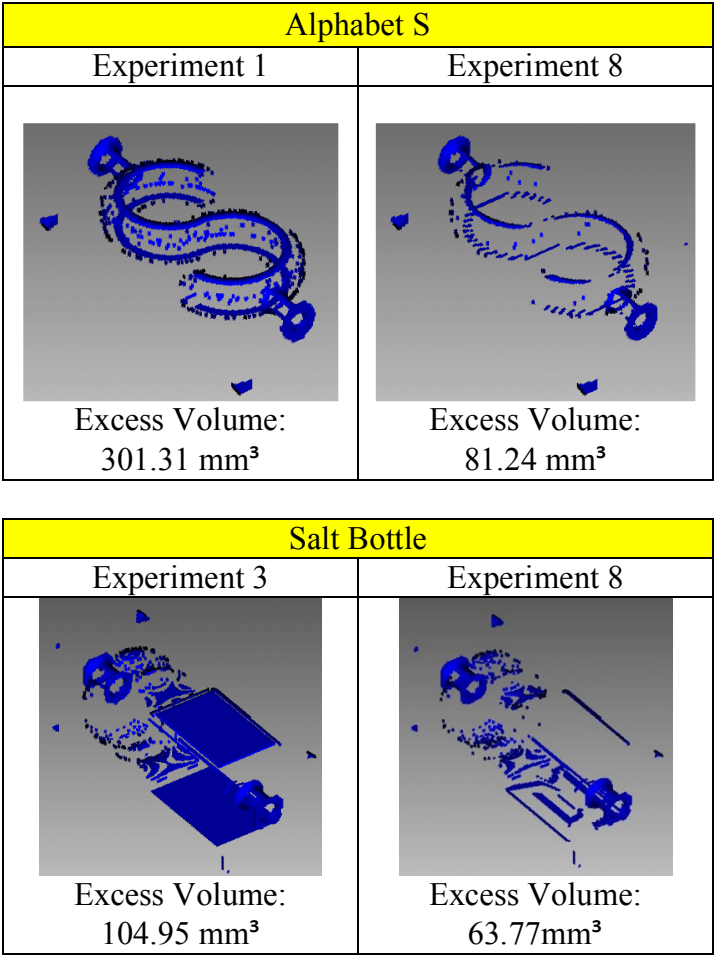

\section{Conclusions}

FinishSimulCAM managed to execute all the machining simulation with minimum process planning accordingly. The developed program provide another solution for machining simulation routines in NX-CAM environment by integrating Visual Basic programming tools to optimize the process planning and able to execute multiple simulation routines effectively. In this paper, a new simulation approach was established to investigate the correlation between Radial depth of cut (RDOC) and Axial depth of cut (ADOC) parameter on the total volume removed. The value of RDOC and ADOC was paired inversely in percentage value and other cutting parameter was set to constant. The presented approach simulate 9 machining operation with different depth of cut. Machining simulation results shows that Axial depth of cut (ADOC) is contributed more on the total volume removed compared to Radial depth of cut (RDOC).

\section{Acknowledgements}

We acknowledge with gratitude to Ministry of Higher Education Malaysia for providing a financial support under Fundamental Research Grant Scheme (FRGS)(RDU160130), and Faculty of Manufacturing Engineering, Universiti Malaysia Pahang which realize this research project.

\section{References}

1. Deepak, S. S. K. Applications of Different Optimization Methods for Metal Cutting
Operation - A Review. Res. J. Eng. Sci. 1, 52-58 (2012).

2. Anderberg, S. A study of process planning for metal cutting. (Chalmers University of Technology, 2009).

3. Wei, S., Chong, L., Jing, Y., Ying, W. \& Junjie, G. A digital management system of part processing parameter based on UG. 2014 IEEE Int. Conf. Mechatronics Autom. IEEE ICMA 2014 2055-2059 (2014). doi:10.1109/ICMA.2014.6886020

4. Palekar, J. \& Khairnar, H. P. Master Model Automation Using NX Unigraphics Customization. Int. J. Eng. Dev. Res. 2, 2184 2189 (2014).

5. Zbiciak, M., Grabowik, C. \& Janik, W. An automation of design and modelling tasks in NX Siemens environment with original software generator module. IOP Conf. Ser. Mater. Sci. Eng. 95, (2015).

6. Gawai, U. ., Wavale, S. . \& Katkam, V. . Software Development (Customization) in UG/NX 4.0 for Coupling Module using Knowledge Fusion Programming. Int. J. Sci. Eng. Technol. Res. 04, 1421-1426 (2015).

7. Siemens. Knowledge Fusion (KF). (2018). Available at: http://www.daasolutions.com/expertise/nxcusto mization/knowledgefusion/. (Accessed: 1st May 2018)

8. Siddesh, S. \& Suresh, B. S. Automation of Generating CAD Models. 5, 55-58 (2015).

9. Benaouali, A. \& Kachel, S. An Automated CAD/CAE Integration System for the Parametric Design of Aircraft Wing Structures. J. Theor. Appl. Mech. 55, 447-459 (2017).

10. Nafis, M., Case, K. \& Watts, D. Rapid process planning in CNC machining for rapid manufacturing applications. Int. J. Mech. Eng. Robot. Res. 6, 118-121 (2017).

11. McKeown, J. Programming in Visual Basic 2010. United States of America by Cambridge University Press, New York (Cambridge University Press, 2010). doi:10.1145/1921532.1921549

12. Hafiz, M. \& Nafis, O. Z. Investigation of roughing machining simulation by using visual basic programming in NX CAM system. IOP Conf. Ser. Mater. Sci. Eng. 319, 12010 (2018).

13. CGTech. VERICUT 8.0: Release Notes. (2016). 